\title{
High plasma levels of oxidatively modified low-density lipoproteins are associated with the suppressed expression of immunomodulatory molecules in patients with hematological malignancies
}

\author{
HAI-QING YANG ${ }^{1}$, FA-QI QIU ${ }^{1}, \mathrm{KE} \mathrm{JIN}^{1}$, NENG-GANG JIANG ${ }^{2}$ and LI ZHANG $^{3}$ \\ ${ }^{1}$ Department of Hematology, Sichuan Academy of Medical Sciences and Sichuan Provincial People's Hospital, \\ Chengdu, Sichuan 610072; ${ }^{2}$ Department of Clinical Laboratories, Ministry of Health; ${ }^{3}$ Laboratory of Pathology, \\ West China Hospital, Sichuan University, Chengdu, Sichuan 610041, P.R. China
}

Received June 5, 2014; Accepted March 17, 2015

DOI: $10.3892 /$ etm.2015.2392

\begin{abstract}
Dyslipidemia is a common feature in immunosuppressed patients, such as kidney and bone marrow transplantation recipients and patients with breast, prostate or gynecological carcinoma or acute lymphoblastic leukemia. In addition, high levels of oxidatively modified low-density lipoproteins (oxLDLs) are closely associated with carcinogenesis. There are, however, no reports on the association between the serum oxLDL levels and the expression of important immunomodulatory molecules in patients with hematological disorders. In the present study, 39 patients with hematological disorders were stratified into four groups: Two groups with malignancies [chronic myeloid leukemia (CML) and acute myeloblastic leukemia (AML)] and two groups without malignancies [myelodysplastic syndrome (MDS) and iron deficiency anemia (IDA)]. Immunomodulatory molecules were monitored in these groups. The enzyme-linked immunosorbent assay results indicated that the plasma oxLDL levels were significantly higher in patients with AML or CML than those in patients with MDS or IDA. The quantitative polymerase chain reaction results revealed that the expression of numerous important immunomodulatory elements, including tumor-related genes, immunological and inflammatory cytokines, defense-responsive genes, genes regulating cell proliferation, adhesion and migration molecules and leukocyte chemotaxis genes, showed considerable variation in patients with hematological disorders, particularly in those with MDS or IDA, as compared with the expression in the healthy volun-
\end{abstract}

Correspondence to: Dr Li Zhang, Laboratory of Pathology, West China Hospital, Sichuan University, 37 Guo Xue Xiang, Chengdu, Sichuan 610041, P.R. China

E mail: zhangli7375@scu.edu.cn

Key words: dyslipidemia, oxidatively modified low-density lipoprotein, hematological malignancy, hematological disorder, immunomodulatory molecules teers. The present study demonstrated that, in patients with a hematological malignancy (either AML or CML), the activation of numerous immune response-related molecules was inhibited. Thus, an association between hematological malignancies and dyslipidemia, i.e. high levels of oxLDL, is suggested. Further research is necessary to investigate how oxLDL influences cancer progression.

\section{Introduction}

It is common for immunosuppressed patients, such as kidney (1) and hematopoietic stem cell transplantation recipients (2), to exhibit dyslipidemia. Such patients are likely to develop lipoprotein modifications that have an atherogenic profile. It has previously been indicated that mortality rates from cardiovascular disease are up to 10-fold greater among immunosuppressed patients than among the normal population (1). Guidelines for the management of dyslipidemia recommend a low-fat diet, exercise and body weight reduction, as well as reductions in alcohol consumption and smoking (3). Dyslipidemia is also commonly found in patients with malignancies. Total cholesterol and low-density lipoprotein (LDL) levels among patients with breast cancer have been shown to be significantly elevated as compared with those in controls (4). Dyslipidemia has also been observed in patients with esophageal carcinoma (5), chronic myeloid leukemia (CML) (6) or acute myeloblastic leukemia (AML) (7). It has been proposed that lipoproteins in the blood influence carcinogenesis, as a result of their fundamental role in the maintenance of cell integrity (8). The exact mechanisms through which lipoproteins may contribute to carcinogenesis remain unidentified.

A previous study indicated that levels of oxidatively modified LDLs (oxLDLs) are elevated in the plasma of patients with acute myocardial infarction as compared with those in healthy individuals (9). The initiation of atherosclerosis is mainly triggered by endothelial dysfunction or activation elicited by oxLDL (10). oxLDL exerts numerous biological activities that are crucial for atherogenesis, including inducing the migration, proliferation and transformation of cells by impairing 
the production of nitric oxide, activating $\mathrm{T}$ lymphocytes and inducing endothelial-leukocyte adhesion molecules, smooth muscle growth factors and proatherogenic genes $(11,12)$. oxLDL-induced activated monocytes upregulate transforming growth factor (TGF- $\beta$ ) expression and increase disease progression (13). In addition, the expression of lectin-like oxidized LDL receptor 1 in endothelial cells and monocytes has a critical function in plaque formation, progression and thrombosis (14). These aforementioned effects lead to fibrosis of the intimal vessels.

To date, the majority of studies on hyperlipidemia have been performed in immunosuppressed patients who have undergone a transplant. Although previous reports have revealed a close association between oxLDL and the malignancy status, there is a missing link between variations in the oxLDL and cytokine/chemokine expression levels for different hematological diseases. We hypothesized that the plasma levels of oxLDL in patients with hematological malignancies are different from those in patients with hematological diseases. We additionally hypothesized that differences in oxLDL levels influence the expression of cytokines, growth factors and inflammatory molecules, which play an important role in inflammatory responses. To confirm the above hypothesis, the aim of the present study was to evaluate the association between the plasma oxLDL levels and the expression of cytokines, chemokines and growth factors in 39 patients and 9 healthy individuals. The patients were stratified into groups according to the type of hematological disorder, namely CML, AML, myelodysplastic syndrome (MDS) or iron deficiency anemia (IDA).

\section{Materials and methods}

Patients. The present study included 39 adult patients with hematological disorders or malignancies. Patients who were considered to have a hematological disorder included those patients with $\operatorname{MDS}(n=8)$ or IDA $(n=11)$, whereas patients who were considered to have a hematological malignancy included those patients with AML $(n=9)$ or CML $(n=11)$. All patients received the first diagnosis based on clinical and laboratory features. Blood smears of their bone marrow were extracted in the Department of Hematology of Sichuan Provincial People's Hospital (Chengdu, China) between 2009 and 2012.

Nine normal healthy controls were selected from a community screening examination in Chengdu, China. Informed consent was obtained from all subjects. The study protocol was approved by the local Ethics Review Committee.

Enzyme-linked immunosorbent assay (ELISA) for oxLDL. The plasma of the patients and normal controls was prepared through centrifugation $(600 \mathrm{x} \mathrm{g}, 5 \mathrm{~min})$ of the fresh blood samples. An ELISA for oxLDL was performed with an oxLDL kit (Diagnostic Systems Laboratories, Inc., Webster, TX, USA). Plasma was diluted 1:1 and incubated in 96-well oxLDL-coated microtitration strips for $30 \mathrm{~min}$ at room temperature, in accordance with the manufacturer's instructions. The plates were then washed three times and incubated with horseradish peroxidase for $30 \mathrm{~min}$ at room temperature. Once the unbound conjugates had been removed by washing the samples three times, tetramethylbenzidine was added to the wells as a chromogenic substrate. The mixture was incubated for $10 \mathrm{~min}$ at room temperature in the dark. A stop solution was used to terminate color development and the absorbance was measured at $450 \mathrm{~nm}$ within $30 \mathrm{~min}$. A standard curve was constructed, using the standards included in the kits, for the purpose of calculating the oxLDL titer. The oxLDL concentration in the samples was quantified in biomedical units, as defined by the manufacturer.

Quantitative polymerase chain reaction ( $q P C R$ ) analysis. Total RNA was extracted using RNeasy Mini kit (Qiagen, Düsseldorf, Germany). cDNA was synthesized using ReverTra Ace ${ }^{\circledR}$ qPCR RT kit (TOYOBO, Kagoshima, Japan), and the reverse transcription conditions were $65^{\circ} \mathrm{C}$ for $5 \mathrm{~min}$, followed by $37^{\circ} \mathrm{C}$ for $15 \mathrm{~min}$ and $98^{\circ} \mathrm{C}$ for $5 \mathrm{~min}$. The qPCR was conducted using RealMaster Mix (SYBR Green) [cat. no. FP202-01; Tiangen Biotech (Beijing) Co., Ltd., Beijing, China] in a Bio-Rad iCycler $\mathrm{iQ}^{\mathrm{TM}}$ Optical Module (584BR; Bio-Rad, Hercules, CA, USA) with the following conditions: One cycle of $95^{\circ} \mathrm{C}$ for $30 \mathrm{sec} ; 40$ cycles of $95^{\circ} \mathrm{C}$ for $30 \mathrm{sec}$, $58^{\circ} \mathrm{C}$ for $30 \mathrm{sec}$ and $72^{\circ} \mathrm{C}$ for $30 \mathrm{sec}$; followed by 80 repeats of $55^{\circ} \mathrm{C}$ for $10 \mathrm{sec}$ to collect melting curve data. The primers used for the PCR assays are listed in Table I. GAPDH was used as an internal control. Bio-Rad iQ5 software was used for the analysis of the qPCR data, and GraphPad Prism 5 software (GraphPad Software, Inc., San Diego, CA, USA) was used for the construction of the figures.

Statistical analysis. All statistical analyses were performed using SPSS 16.0 (SPSS Inc., Chicago, IL, USA). The characteristics of the patients are presented as the mean \pm standard deviation. The t-test was used to perform quantitative data analysis. $\mathrm{P} \leq 0.05$ was considered to indicate a statistically significant difference. The data obtained from healthy volunteers were used as the control data.

\section{Results}

Patient characteristics. Table II shows the clinical and biochemical characteristics of the patients that took part in the study. No significant differences were found in the systolic blood pressure or the red blood cell, urea, total protein, albumin, alanine aminotransferase, mean corpuscular volume and mean corpuscular hemoglobin levels of the patients; however, the gender distribution, age, diastolic blood pressure and white blood cell, neutrophil, lymphocyte, hemoglobin, platelet, creatine, aspartate aminotransferase and $\gamma$-glutamyl transpeptidase levels were significantly different among the five groups.

Comparison of the serum oxLDL levels among the five groups. The ELISA results showed that the plasma oxLDL levels differed among the groups, with significant elevations in the AML and CML groups compared with the control group $(\mathrm{P}<0.001)$. The average plasma oxLDL levels in the AML and CML groups were 350 and $260 \mu \mathrm{g}$, respectively, while in the MDS and IDA groups the average levels were 120 and $130 \mu \mathrm{g}$, respectively, which were comparable with the levels in the normal controls (Fig. 1). 
Table I. Oligonucleotides used for the quantitative polymerase chain reaction.

\begin{tabular}{|c|c|c|c|}
\hline Gene & Forward primer $\left(5^{\prime}-3^{\prime}\right)$ & Reverse primer $\left(5^{\prime}-3^{\prime}\right)$ & $\begin{array}{c}\text { GenBank } \\
\text { number }\end{array}$ \\
\hline IL-5 & AGCTGCCTACGTGTATGCCA & GTGCCAAGGTCTCTTTCACCA & J03478 \\
\hline IL-6 & GACCCAACCACAAATGCCA & GTCATGTCCTGCAGCCACTG & M14584 \\
\hline IL-10 & GGTGATGCCCCAAGCTGA & TCCCCCAGGGAGTTCACA & U16720 \\
\hline IL-11 & CGAGCGGACCTACTGTCCTA & GCCCAGTCAAGTGTCAGGTG & NM_000641 \\
\hline IL-12 & CGGTCATCTGCCGCAAA & CAAGATGAGCTATAGTAGCGGTCCT & M65272 \\
\hline IL-15 & GACCCCACCAAAGCTGGAC & TCACAGTGCTGCTGTCTGCTG & M90391 \\
\hline IFN- $\alpha$ & GGTGCTCAGCTGCAAGTCAA & GCTACCCAGGCTGTGGGTT & J00207 \\
\hline IFN- $\beta$ & CAGCAATTTTCAGTGTCAGAAGCT & TCATCCTGTCCTTGAGGCAGT & M28622 \\
\hline IFN- $\gamma$ & CCAACGCAAAGCAATACATGA & CGCTTCCCTGTTTTAGCTGC & J00219 \\
\hline TGF- $\beta$ & TATCGACATGGAGCTGGTGAAG & CAGCTTGGACAGGATCTGGC & $\mathrm{X} 02812$ \\
\hline TNF- $\alpha$ & GGTGCTTGTTCCTCAGCCTC & CAGGCAGAAGAGCGTGGTG & M10988 \\
\hline iNOS & CCAACAATGGCAACATCAGG & TCGTGCTTGCCATCACTCC & L09210 \\
\hline IP-10 & TGAAATTATTCCTGCAAGCCAA & CAGACATCTCTTCTCACССТTCTTT & NM_001565 \\
\hline MIP-1 $1 \alpha$ & AGCTGACTACTTTGAGACGAGCAG & CGGCTTCGCTTGGTTAGGA & NM_002983 \\
\hline MIP-1 $\beta$ & CTGCTCTCCAGCGCTCTCA & GTAAGAAAAGCAGCAGGCGG & NM_002984 \\
\hline RANTES & GACACCACACCCTGCTGCT & TACTCCTTGATGTGGGCACG & NM_002985 \\
\hline MCP-2 & GTTTCTGCAGCGCTTCTGTG & TGGCTGAGCAAGTCCCTGA & Y10802 \\
\hline MCP-3 & AGCAGAGGCTGGAGAGCTACA & GGGTCAGCACAGATCTCCTTGT & NM_006273 \\
\hline p53 & CTTGCATTCTGGGACAGCCAAG & CACGCAAATTTCCTTCCACTCGG & DQ892492 \\
\hline $\operatorname{cdc} 2$ & CAGGTTATATCTCATCTTTGAG & GTTGAGTAACGAGCTGACCCC & AM393287 \\
\hline ITBG9 & GCAGTGACCGCTGGCCACTT & GCGCACAAGGAGGAGCCGAA & NM_002207.2 \\
\hline ICAM-1 & CGAGCTTGGCTGTGGCCTCC & TCTCCGCCATCCCAGCCTCC & NR_002947.1 \\
\hline VCAM-1 & AGGTGACGAATGAGGGGACCACA & CCAGCCTCCAGAGGGCCACT & NM_001078.3 \\
\hline CCL21 & TGGTTCTGGCCTTTGGCA & AGGCAACAGTCCTGAGCCC & NM_002989 \\
\hline CCL24 & AGCCTTCTGTTCCTTGGTGTCT & GGGAGAGGGTATGACCACAGAG & NM_002991 \\
\hline CCL25 & CAGGAAGGTGTGTGGGAACC & CGAGCATCCAGGAGCTTCA & NM_005624 \\
\hline CCL27 & GAGCCCAGACCCTACAGCAG & GGCTTTCGGTAGAGCTGAGTACA & NM_006664 \\
\hline GAPDH & GAAGGTGAAGGTCGGAGTC & GAAGATGGTGATGGGATTTC & $\mathrm{J} 04038$ \\
\hline
\end{tabular}

IL, interleukin; IFN, interferon; TGF, transforming growth factor; TNF, tumor necrosis factor; iNOS, inducible nitric oxide synthase; IP-10, $10 \mathrm{kDa}$ interferon $\gamma$-induced protein; MIP, macrophage inflammatory protein; RANTES, regulated on activation normal T-cell expressed and secreted; MCP, monocyte chemotactic protein; cdc2, cyclin-dependent kinase 1; ITBG, integrin $\beta$; ICAM, intercellular adhesion molecule; VCAM, vascular cell adhesion molecule; CCL, chemokine (C-C motif) ligand.

Expression of cancer-related genes and chemokines in patients with hematological disorders. In order to determine the association between the oxLDL level and gene expression variation, qPCR was conducted. The qPCR results indicated changes in the expression of genes associated with the following biological processes: i) Cell cycle, ii) immune and inflammatory responses, iii) defense response to viruses and other pathogens, iv) regulation of cell proliferation, v) regulation of cell migration, vi) leukocyte chemotaxis and vii) cell adhesion.

p53 is a well-known anticancer gene. Suppressed expression of p53 was found in all patients, as compared with the normal controls $(\mathrm{P}<0.001)$ (Fig. 2). By contrast, the expression of the cell cycle gene cyclin-dependent kinase 1 (cdc2) was significantly higher in patients with hematological disorders than that in the normal controls $(\mathrm{P}<0.001)$. These results indicated increased cell proliferation in those patients (Fig. 2).
Notably, the detection of chemokine expression showed the overexpression of chemokine (C-C motif) ligand-21 (CCL-21), CCL-24, CCL-25 and CCL-27 in patients with MDS or IDA $(\mathrm{P}<0.001)$, while only a slight increase was observed in patients with AML or CML $(\mathrm{P}<0.05)$ (Fig. 2). These results suggest that high levels of oxLDL suppress the proinflammatory response in disease states.

Variation in the gene expression of interferons (IFNs) and adhesion molecules. IFNs play an important role in the host defense against viruses and other pathogens. Upon analysis of the three major members of this family, IFN- $\alpha$, $-\beta$ and $-\gamma$, higher levels of IFN- $\alpha$ and $-\beta$ were found in the patients with hematological disorders as compared with the normal controls $(\mathrm{P}<0.05)$ (Fig. 3). The level of IFN- $\gamma$ was observed to be increased only in the MDS and IDA groups $(\mathrm{P}<0.05)$ (Fig. 3). 
Table II. Characteristics of the patients and normal controls in the present study.

\begin{tabular}{|c|c|c|c|c|c|}
\hline Characteristics & AML, $n=9$ & CML, $n=11$ & MDS, $n=8$ & IDA, $n=11$ & $\mathrm{NL}, \mathrm{n}=9$ \\
\hline Gender (male/female) & $3 / 6$ & $4 / 7$ & $7 / 1$ & $2 / 9$ & $5 / 4$ \\
\hline Age $\left(\right.$ years) ${ }^{\mathrm{a}}$ & $53.6 \pm 16.0$ & $46.3 \pm 20.2$ & $47.8 \pm 21.2$ & $47.3 \pm 19.7$ & $38.4 \pm 10.2$ \\
\hline $\mathrm{SBP}(\mathrm{mmHg})^{\mathrm{a}}$ & $123.2 \pm 28.9$ & $108 \pm 10.6$ & $131.6 \pm 23.6$ & $113.0 \pm 12.3$ & $112.4 \pm 9.6$ \\
\hline $\mathrm{DBP}(\mathrm{mmHg})^{\mathrm{a}}$ & $69.5 \pm 16.6$ & $62.6 \pm 8.8$ & $81.4 \pm 16.3$ & $65.9 \pm 9.9$ & $78.3 \pm 6.9$ \\
\hline Hypertension (Y/N) & $1 / 8$ & $0 / 11$ & $2 / 8$ & $1 / 11$ & $0 / 9$ \\
\hline WBC $\left(10^{9} / 1\right)^{\mathrm{a}}$ & $33.4 \pm 11.6$ & $25.5 \pm 4.8$ & $14.8 \pm 1.3$ & $7.4 \pm 5.6$ & $7.5 \pm 2.3$ \\
\hline Neutrophil (\%) & $49.5 \pm 29.8$ & $57.9 \pm 28.2$ & $67.9 \pm 20.9$ & $76.1 \pm 15.9$ & $65.8 \pm 6.7$ \\
\hline Lymphocyte $(\%)^{\mathrm{a}}$ & $47.9 \pm 22.1$ & $26.2 \pm 11.2$ & $12.6 \pm 6.7$ & $15.8 \pm 5.8$ & $11.4 \pm 2.3$ \\
\hline $\operatorname{RBC}\left(10^{12} / 1\right)^{\mathrm{a}}$ & $2.3 \pm 0.9$ & $3.1 \pm 0.8$ & $2.1 \pm 0.3$ & $3.1 \pm 1.8$ & $2.6 \pm 1.1$ \\
\hline $\mathrm{Hb}(\mathrm{g} / \mathrm{l})^{\mathrm{a}}$ & $65.6 \pm 29.1$ & $79.2 \pm 29.3$ & $53.4 \pm 4.4$ & $67.9 \pm 25.2$ & $68.2 \pm 6.7$ \\
\hline $\operatorname{PLT}\left(10^{9} / 1\right)^{\mathrm{a}}$ & $50.6 \pm 6.6$ & $77.9 \pm 58.8$ & $48.1 \pm 57.5$ & $203.6 \pm 22.1$ & $69.3 \pm 12.2$ \\
\hline Urea $(\mathrm{mM})^{\mathrm{a}}$ & $4.9 \pm 0.8$ & $5.2 \pm 1.9$ & $5.8 \pm 3.0$ & $5.3 \pm 4.4$ & $4.6 \pm 0.8$ \\
\hline Creatine $(\mu \mathrm{M})^{\mathrm{a}}$ & $70.1 \pm 10.0$ & $53.3 \pm 23.6$ & $83.8 \pm 33.1$ & $64.5 \pm 12.4$ & $65.3 \pm 22.3$ \\
\hline $\mathrm{TP}(\mathrm{g} / \mathrm{l})^{\mathrm{a}}$ & $56.5 \pm 4.8$ & $61.7 \pm 11.6$ & $65.9 \pm 14.7$ & $62.7 \pm 7.5$ & $61.8 \pm 5.7$ \\
\hline $\operatorname{ALB}(\mathrm{g} / \mathrm{l})^{\mathrm{a}}$ & $38.2 \pm 3.4$ & $37.7 \pm 7.3$ & $38.7 \pm 14.2$ & $37.1 \pm 6.2$ & $36.2 \pm 5.2$ \\
\hline $\operatorname{AST}(\mathrm{U} / 1)^{\mathrm{a}}$ & $23.5 \pm 10.4$ & $62.1 \pm 7.8$ & $28.0 \pm 16.6$ & $15.8 \pm 7.8$ & $19.4 \pm 2.3$ \\
\hline $\operatorname{ALT}(\mathrm{U} / 1)^{\mathrm{a}}$ & $25.0 \pm 10.9$ & $29.9 \pm 17.1$ & $25.5 \pm 16.7$ & $25.8 \pm 7.8$ & $25.1 \pm 5.7$ \\
\hline GGT $(\mathrm{U} / 1)^{\mathrm{a}}$ & $93.7 \pm 11.4$ & $50.3 \pm 8.3$ & $40.0 \pm 30.1$ & $14.5 \pm 7.1$ & $22.5 \pm 5.6$ \\
\hline $\operatorname{MCV}(\mathrm{fl})^{\mathrm{a}}$ & $97.0 \pm 4.4$ & $87.3 \pm 7.9$ & $93.4 \pm 8.6$ & $84.9 \pm 21.5$ & $87.2 \pm 15.3$ \\
\hline $\mathrm{MCH}(\mathrm{pg})^{\mathrm{a}}$ & $32.7 \pm 2.2$ & $27.8 \pm 3.7$ & $31.0 \pm 3.5$ & $32.5 \pm 2.5$ & $31.6 \pm 8.3$ \\
\hline
\end{tabular}

${ }^{\text {aPresented }}$ as the mean \pm standard deviation. SBP, systolic blood pressure; DBP, diastolic blood pressure; WBC, white blood cell; N, no; Y, yes; RBC, red blood cell; Hb, hemoglobin; PLT, platelet; TP, total protein; ALB, albumin; AST, aspartate aminotransferase; ALT, alanine aminotransferase; GGT, $\gamma$ glutamyl transpeptidase; MCV, mean corpuscular volume; $\mathrm{MCH}$, mean corpuscular hemoglobin; AML, acute myeloblastic leukemia; CML, chronic myeloid leukemia; MDS, myelodysplastic syndrome; IDA, iron deficiency anemia; NL, normal control.

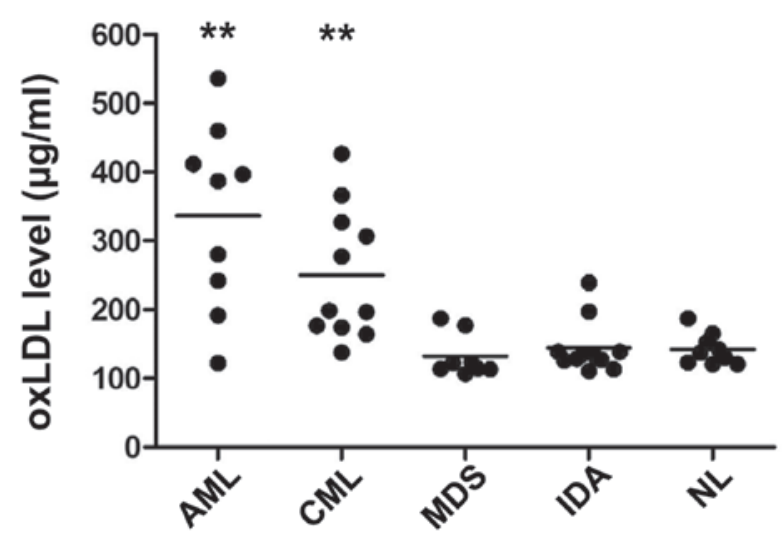

Figure 1. Plasma oxLDL levels in patients with hematological disorders. ${ }^{* *} \mathrm{P}<0.001$ versus the control group. oxLDL, oxidatively modified low-density lipoprotein; AML, acute myeloblastic leukemia; CML, chronic myeloid leukemia; MDS, myelodysplastic syndrome; IDA, iron deficiency anemia; NL, normal control.

With regard to the cell adhesion molecules, the expression of the three most important families [intercellular adhesion molecules (ICAMs), vascular cell adhesion molecules (VCAMs) and integrin $\beta$ (ITGB) 9] was assessed. When compared with the expression in healthy volunteers, the expression of VCAM-1 was downregulated in patients with tumors $(\mathrm{P}<0.05)$, while ITGB9 and ICAM-1 expression was increased in patients with AML or CML (Fig. 3).
Gene expression of interleukins (ILs) is increased in patients with non-malignant disorders. ILs play a key role in inflammatory responses, immunomodulation and cell proliferation. It was observed that, while IL-10 and IL-15 expression in patients with AML or CML remained the same as that in the normal controls, the expression of IL-11 was reduced $(\mathrm{P}<0.05)$ (Fig. 4). The expression levels of IL-6, IL-12 and IL-5 were slightly increased in patients with AML or CML $(\mathrm{P}<0.05)$ (Fig. 4) compared with levels in the controls. Based on this observation, we hypothesized that the activation of IL-related immune responses is suppressed in patients with hematological carcinomas.

Gene expression of growth factors and macrophage chemotaxis proteins. Compared with the control expression levels, the expression of tumor necrosis factor- $\alpha$ (TNF- $\alpha$ ) was found to be significantly upregulated in the MDS and IDA groups $(\mathrm{P}<0.001)$, while it was slightly increased in the AML and CML groups $(\mathrm{P}<0.05)$. The TGF- $\beta$ expression in the AML $(\mathrm{P}<0.05)$ and $C M L(P<0.001)$ groups was greater than that in the controls, while in the MDS and IDA groups it remained at a normal level. The expression levels of monocyte chemotactic protein-2 (MCP-2), macrophage inflammatory protein-1 $\beta$ (MIP-1 $\beta$ ) and inducible nitric oxide synthase (iNOS) were increased markedly in patients without tumors $(\mathrm{P}<0.001)$, while a minor upregulation was observed in those with malignancies $(\mathrm{P}<0.05)$. The MCP-3 expression levels were only 


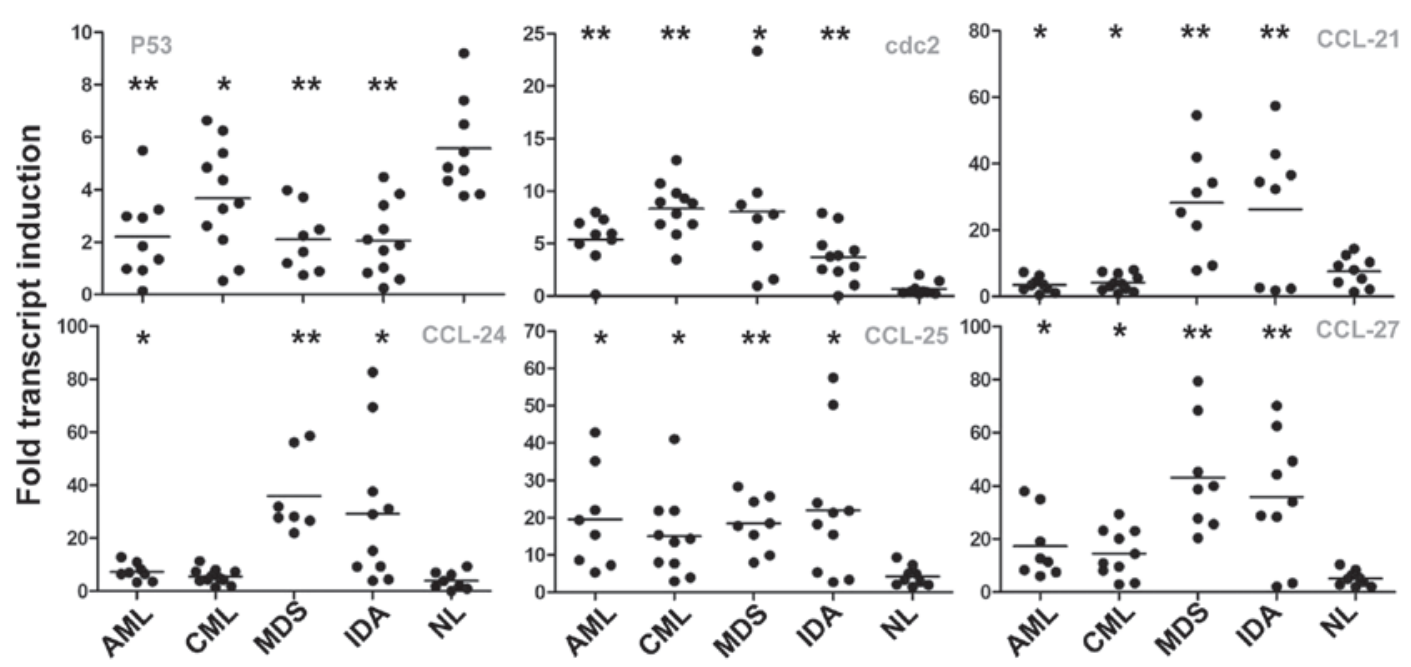

Figure 2. Gene expression variation I: Cancer-related genes and chemokines. ${ }^{*} \mathrm{P}<0.05$ and ${ }^{* *} \mathrm{P}<0.001$ versus the control group. AML, acute myeloblastic leukemia; CML, chronic myeloid leukemia; MDS, myelodysplastic syndrome; IDA, iron deficiency anemia; NL, normal control; cdc2, cyclin-dependent kinase 1; CCL, chemokine (C-C motif) ligand.

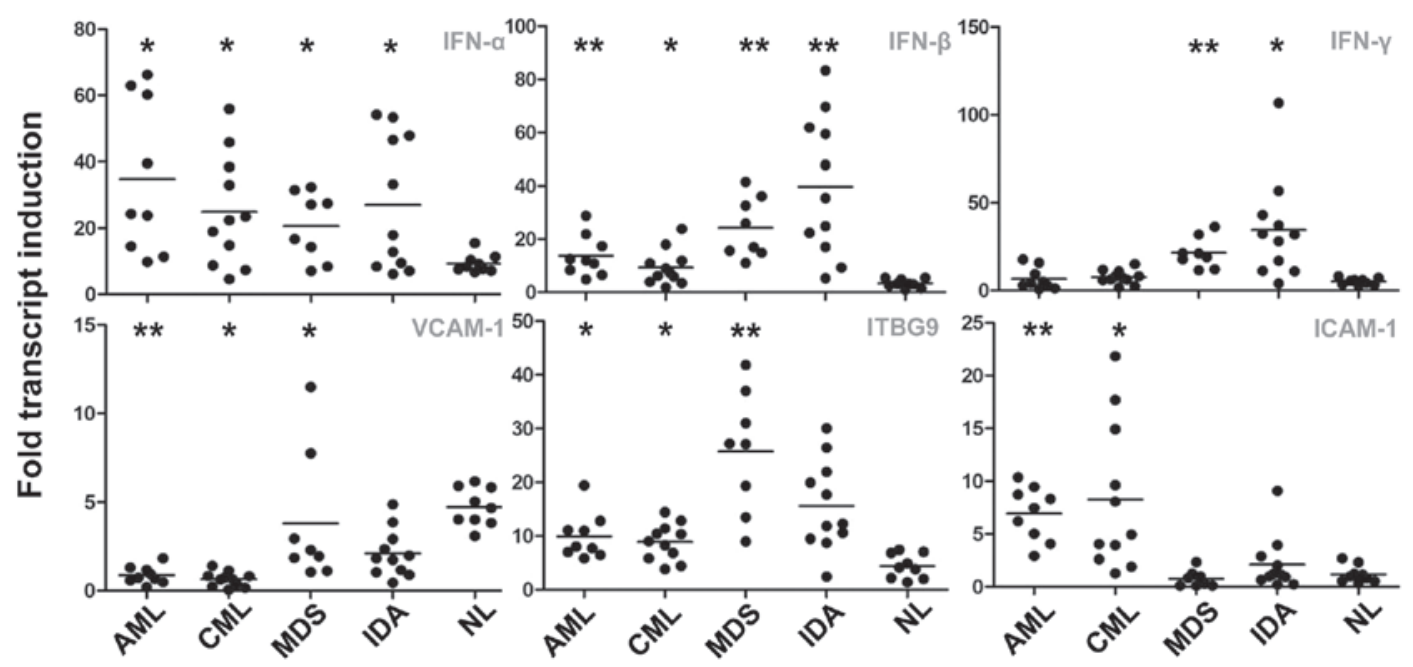

Figure 3. Gene expression variation II: IFNs and adhesion molecules. " $\mathrm{P}<0.05$ and ${ }^{* *} \mathrm{P}<0.001$ versus the control group. AML, acute myeloblastic leukemia; CML, chronic myeloid leukemia; MDS, myelodysplastic syndrome; IDA, iron deficiency anemia; NL, normal control; IFN, interferon; VCAM, vascular cell adhesion molecule; ICAM, intercellular adhesion molecule; ITBG, integrin $\beta$.

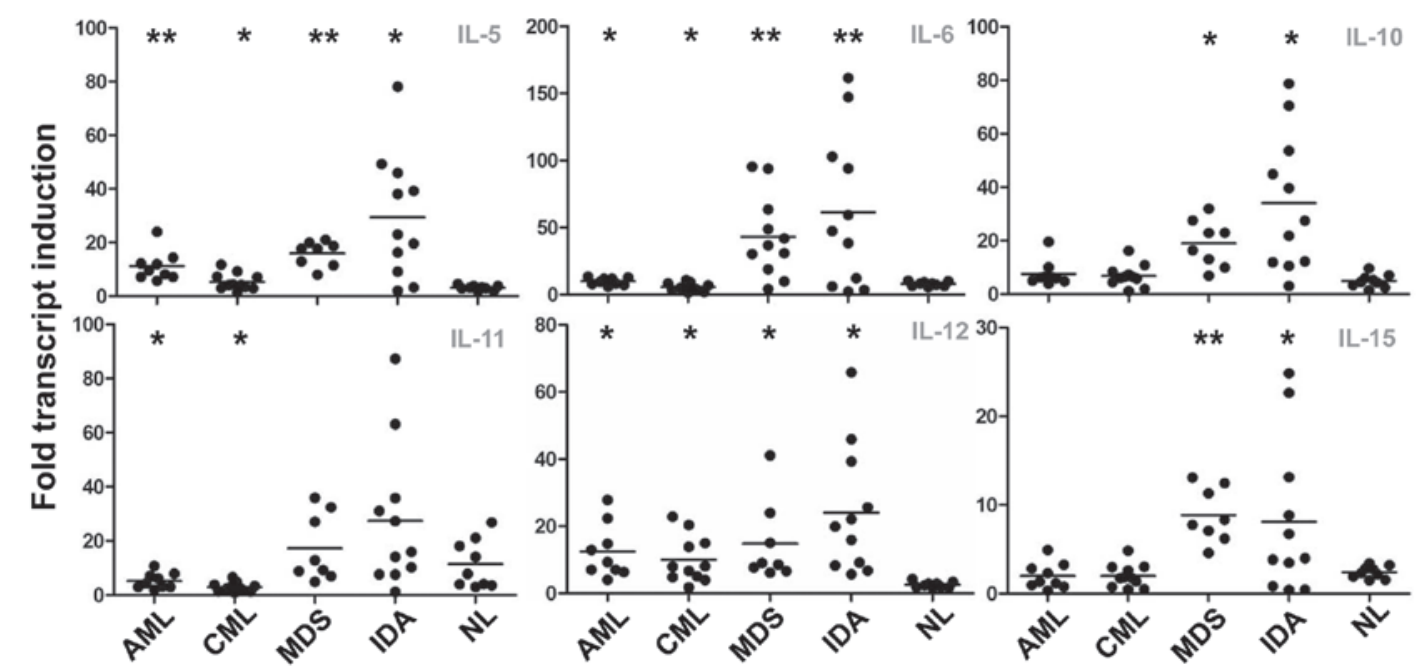

Figure 4. Gene expression variation III: ILs. ${ }^{*} \mathrm{P}<0.05$ and ${ }^{* *} \mathrm{P}<0.001$ versus the control group. AML, acute myeloblastic leukemia; CML, chronic myeloid leukemia; MDS, myelodysplastic syndrome; IDA, iron deficiency anemia; NL, normal control; IL, interleukin. 


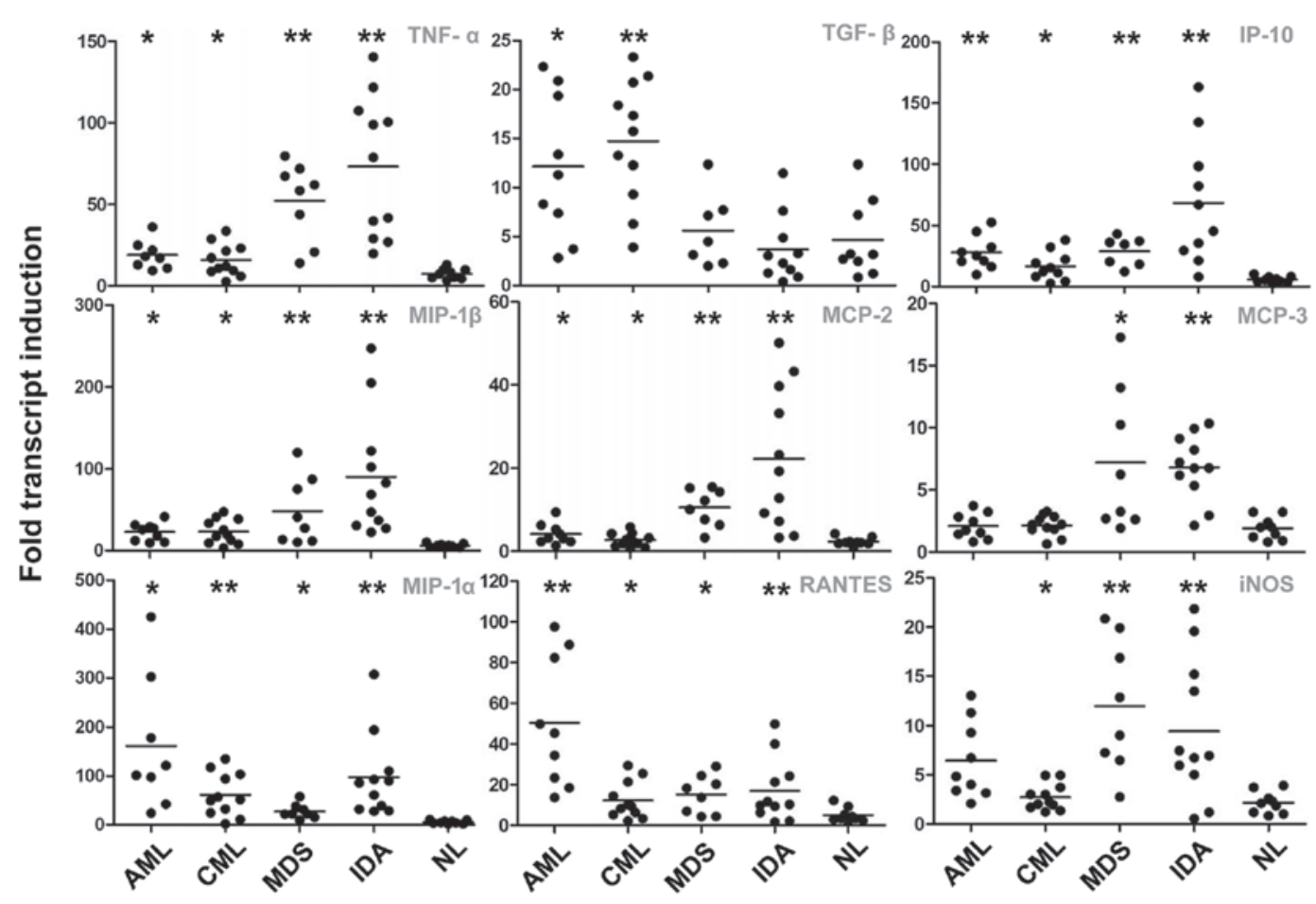

Figure 5. Gene expression variation IV: Growth factors and macrophage chemotactic proteins. ${ }^{*} \mathrm{P}<0.05$ and ${ }^{* *} \mathrm{P}<0.001$ versus the control group. AML, acute myeloblastic leukemia; CML, chronic myeloid leukemia; MDS, myelodysplastic syndrome; IDA, iron deficiency anemia; NL, normal control; TNF, tumor necrosis factor; TGF, transforming growth factor; IP-10, $10 \mathrm{kDa}$ interferon $\gamma$-induced protein; MIP, macrophage inflammatory protein; MCP monocyte chemotactic protein; RANTES, regulated on activation normal T-cell expressed and secreted; iNOS, inducible nitric oxide synthase.

increased in the MDS $(\mathrm{P}<0.05)$ and IDA $(\mathrm{P}<0.001)$ groups. MIP-1 $\alpha, 10 \mathrm{kDa}$ IFN- $\gamma$-induced protein and regulated on activation normal T-cell expressed and secreted expression was similarly upregulated in all the patient groups (Fig. 5).

\section{Discussion}

Hyperlipidemia is a common and serious problem among adult renal and bone marrow stem cell transplantation recipients and patients with malignancies, and it has been demonstrated that the condition contributes to an increase in the risk of cardiovascular diseases and elevated morbidity and mortality within these populations (15). Previous research has demonstrated an association between plasma/serum lipoproteins and different types of cancers, such as breast, prostate and gynecological cancer and acute lymphoblastic leukemia (8). The main question is whether dyslipidemia predisposes one to cancer or whether it is an effect of the malignancy.

Previous studies have found that oxLDL plays an important role in various types of malignancies $(16,17)$. oxLDL expression has been shown to decrease during the progression of esophageal cancer (16), while Petridou et al (17) have reported an association between adiponectin expression and childhood myeloblastic leukemia. A study using an animal model has suggested that a high oxLDL level is closely associated with carcinogenesis (18). Although conflicting data have been reported regarding the link between oxLDL level and the risk of cancer-related mortality $(19,20)$, accumulating evidence suggests that oxLDL induces the production of leukocyte adhesion molecules and chemokines and decreases the release of nitric oxide, which is associated with endothelial dysfunc- tion (11). It has also been shown that oxLDL may be involved in atherogenesis and atherothrombosis by monocyte activation and the accumulation of foam cells (21).

Cytokines and chemokines, as well as numerous other growth factors and adhesion molecules, play an important role in carcinoma progression and inflammatory responses. The progression of carcinomas is closely associated with the unusual expression of several cytokines and growth factors; however, there are no reports regarding the link between plasma oxLDL levels and cytokine/chemokine expression in patients with hematological disorders. In the present study, patients were divided into four groups: Two groups with malignancies (AML and CML) and two groups with nonmalignant disorders (MDS and IDA). The findings of the study demonstrated that the plasma oxLDL levels were significantly higher in the AML and CML groups as compared with those in the non-carcinoma groups (MDS and IDA) and the normal controls. This result is consistent with the results of a previous study, in which the oxLDL serum levels in patients with hepatocellular carcinoma and squamous cell carcinoma of the esophagus were lower than those in the normal controls (22).

Under pathological conditions, a number of immune response-related factors are upregulated and secreted in response to the hostile environment. In the qPCR analysis that was performed in the present study, the upregulation of p53 and the downregulation of cdc2 in patients with hematological disorders suggested that these patients had a decreased antitumor effect and increased cell proliferation. The most noteworthy result of this study was that the activation of numerous proinflammatory genes, including CCL-21, CCL-24, CCL-27, 
IFN- $\beta$, IFN- $\gamma$, IL-5, IL-6, IL-10, IL-15, TNF- $\alpha$, MCP-2, MCP-3 and iNOS, was inhibited in the patients with cancer (the AML and CML groups). Among these factors, IFN- $\beta$ and IFN- $\gamma$ are known to mediate antitumor effects, and TNF- $\alpha$ is known to inhibit tumor progression by inducing the apoptosis of cancer cells (23). It remains to be determined whether these differences were due to the dyslipidemic condition.

There is considerable evidence suggesting that lipid peroxidation products, including oxLDL and anti-oxLDL autoantibodies, play a key role in cancer development $(24,25)$. High serum levels of oxLDL could lead to uncontrolled cell proliferation and reduced apoptosis, contributing to carcinogenesis induction. oxLDL increases the activity of protein kinase- $\mathrm{C}$, which is a serine-threonine-kinase that is involved in the cytoplasmic transduction of the mytogenic signaling pathway (26). oxLDL is also able to induce the production and delivery of other growth factors, such as platelet-derived growth factor, possibly through IL-1 and TNF- $\alpha$, thereby regulating tumor growth (27).

A number of contradictory results, such as the downregulation of VCAM-1 and upregulation of ICAM 1, were found in the present study. The same was also found for IL-5, IL-11 and IL-12, in that the results were not consistent in the patients with cancer. It is possible, however that these contradictions were due to the small number of patients in each study group. Another limitation was that only gene expression detection was used. It is possible that protein expression and secretion studies would enhance the results. The results of the current study suggest a close association between high oxLDL levels and the status of patients with hematological disorders. Further research is required to delineate the detailed mechanisms of the oxLDL-mediated regulation of disease.

\section{Acknowledgements}

This study was supported by the National Basic Research Program of China (grant no. 2009CB522401). The authors would like to thank the nursing staff for their support in this clinical study.

\section{References}

1. Chadban S, Chan M, Fry K, et al; CARI: The CARI guidelines. Nutritional management of dyslipidaemia in adult kidney transplant recipients. Nephrology (Carlton) 15 Suppl 1: S62-S67, 2010.

2. Griffith ML, Savani BN and Boord JB: Dyslipidemia after allogeneic hematopoietic stem cell transplantation: Evaluation and management. Blood 116: 1197-1204, 2010.

3. Tonkin A, Barter P, Best J, et al; National Heart Foundation of Australia; Cardiac Society of Australia and New Zealand: National Heart Foundation of Australia and the Cardiac Society of Australia and New Zealand: Position statement on lipid management - 2005. Heart Lung Circ 14: 275-291, 2005.
4. Ray $\mathrm{G}$ and Husain SA: Role of lipids, lipoproteins and vitamins in women with breast cancer. Clin Biochem 34: 71-76, 2001.

5. Diao Y, Li H, Li H, et al: Association of serum levels of lipid and its novel constituents with the different stages of esophageal carcinoma. Lipids Health Dis 8: 48, 2009.

6. Gottardi M, Manzato E and Gherlinzoni F: Imatinib and hyperlipidemia. N Engl J Med 353: 2722-2723, 2005.

7. Li H, Diao YT, Li HQ, et al: The association between serum levels of oxLDL-IgG and oxLDL-IgM autoantibody with adult acute myeloblastic leukaemia. Lipids Health Dis 9: 11, 2010.

8. Bielecka-Dąbrowa A, Hannam S, Rysz J and Banach M: Malignancy-associated dyslipidemia. Open Cardiovasc Med J 5: 35-40, 2011.

9. Tatsuguchi M, Furutani M, Hinagata J, et al: Oxidized LDL receptor gene (OLR1) is associated with the risk of myocardial infarction. Biochem Biophys Res Commun 303: 247-250, 2003.

10. Chen M, Masaki T and Sawamura T: LOX-1, the receptor for oxidized low-density lipoprotein identified from endothelial cells: Implications in endothelial dysfunction and atherosclerosis. Pharmacol Ther 95: 89-100, 2002.

11. Ross R: Atherosclerosis - an inflammatory disease. N Engl J Med 340: 115-126, 1999.

12. Castelló IB: Hyperlipidemia: A risk factor for chronic allograft dysfunction. Kidney Int Suppl: 73-77, 2002.

13. Drüeke TB, Abdulmassih Z, Lacour B, Bader C, Chevalier A and Kreis H: Atherosclerosis and lipid disorders after renal transplantation. Kidney Int Suppl 31: S24-S28, 1991.

14. Mehta JL, Chen J, Hermonat PL, Romeo F and Novelli G: Lectin-like, oxidized low-density lipoprotein receptor-1 (LOX-1): A critical player in the development of atherosclerosis and related disorders. Cardiovasc Res 69: 36-45, 2006.

15. Silverstein DM, Palmer J, Polinsky MS, Braas C, Conley SB and Baluarte HJ: Risk factors for hyperlipidemia in long-term pediatric renal transplant recipients. Pediatr Nephrol 14: 105-110, 2000.

16. Wang Y, Li H, Diao Y, et al: Relationship between oxidized LDL antibodies and different stages of esophageal carcinoma. Arch Med Res 39: 760-767, 2008.

17. Petridou E, Mantzoros CS, Dessypris N, Dikalioti SK and Trichopoulos D: Adiponectin in relation to childhood myeloblastic leukaemia. Br J Cancer 94: 156-160, 2006.

18. Chung FL, Nath RG, Ocando J, Nishikawa A and Zhang L: Deoxyguanosine adducts of t-4-hydroxy-2-nonenal are endogenous DNA lesions in rodents and humans: Detection and potential sources. Cancer Res 60: 1507-1511, 2000.

19. Eichholzer M, Stähelin HB, Gutzwiller F, Lüdin E and Bernasconi F: Association of low plasma cholesterol with mortality for cancer at various sites in men: 17-y follow-up of the prospective Basel study. Am J Clin Nutr 71: 569-574, 2000.

20. Iribarren C, Reed DM, Burchfiel CM and Dwyer JH: Serum total cholesterol and mortality. Confounding factors and risk modification in Japanese-American men. JAMA 273: 1926-1932, 1995.

21. Puccetti L, Pasqui AL, Bruni F, et al: Lectin-like oxidized-LDL receptor-1 (LOX-1) polymorphisms influence cardiovascular events rate during statin treatment. Int J Cardiol 119: 41-47, 2007.

22. Uchida K: 4-Hydroxy-2-nonenal: A product and mediator of oxidative stress. Prog Lipid Res 42: 318-343, 2003.

23. Vicari AP and Caux C: Chemokines in cancer. Cytokine Growth Factor Rev 13: 143-154, 2002

24. Guyton KZ and Kensler TW: Oxidative mechanisms in carcinogenesis. Br Med Bull 49: 523-544, 1993.

25. Wiseman $\mathrm{H}$ and Halliwell B: Damage to DNA by reactive oxygen and nitrogen species: Role in inflammatory disease and progression to cancer. Biochem J 313: 17-29, 1996.

26. Kelly K, Cochran BH, Stiles CD and Leder P: Cell specific regulation in normal and neoplastic cells. Adv Cancer Res 56: 1-48, 1990.

27. Oberhammer F, Bursch W,Parzefall W, et al: Effect of transforming growth factor beta on cell death of cultured rat hepatocytes. Cancer Res 51: 2478-2485, 1991. 